\title{
INTERIOR FINISHING BUILDING ROBOT "TAMIR"
}

\author{
Yehiel Rosenfeld, Abraham Warszawski and Uri Zajicek \\ National Building Research Institute \\ Faculty of Civil Engineering \\ Technion - Israel Institute of Technology \\ Haifa 32000, ISRAEL
}

\begin{abstract}
The latest development in the evolution of TAMIR - Technion Autonomous Multipurpose Interior Robot - is described. The robot performs, at this stage, three types of full scale interior finishing works: a. wall building b. plastering/painting c. tile setting.

The former task was perfected during the last year and it is described in detail in this paper, with regard to (1) Accuray analysis, (2) Design and performance of the end effector, and (3) Callibration and correction of errors.

The present stage is intended to examine the total feasibility of the application in both technological terms (with all peripheral works), and economically, by measurement of robotic vs. manual performance, pertained to a large partition segment with a corner, openings and different edge conditions at its interfaces with adjacent walls, floor and ceiling.
\end{abstract}

\section{INTRODUCTION}

"TAMIR" stands for: "Technion Autonomous Multipurpose Interior Robot", which was developed in recent years by the Israel National Building Research Institute. The concept of a multipurpose interior finishing robot for building applications was suggested by Warszawski $(1984,1990)$, as one member in a family of four generic types of building robots. The other three members are: (1) a large assembly robot, which is also being developed at the Technion (Rosenfeld and Berkowitz 1989, 1990), in the modified form of a semi-automated tower crane; (2) a floor finishing robot, which is now at the feasibility study stage; and (3) a hanging/climbing robot for exterior facades of buildings.

The development of TAMIR - the interior robot - involved several stages and sub-projects: The preliminary study contained a comprehensive analysis of all interior works to determine their suitability for robotic execution, and concluded with an actual proof of concept with the aid of a small educational robot. In ISARC 4 - held in Haifa - 
Warszawski and Argaman (1987) presented, with that robot, three model applications: wall building, spray painting, and the sealing of joints. Important aspects, such as determination of the desired geometrical configuration and the size and expected productivity of a full scale multi-purpose interior robot - had been studied extensively through graphic simulation by Warszawski and Navon (1990), and presented at ISARC 4 and 6. Another parallel study (Argaman and Warszawski 1989) dealt with the planning process of execution of building interior works by a mobile robot, given the layout of a typical floor, the characteristics of the task, and the characteristics of the robot. Meanwhile, two additional projects had been launched: the first, dealing with full size applications of interior tasks with a large mobile robot from a temporary workstation. It was partly presented in ISARC 7 (Rosenfeld, Warszawski and Zajicek 1990), and is continued in this paper. The second, parallel project in this last stage of evolution develops autonomous navigation and mapping and task planning systems for TAMIR.

The present paper is an in-depth study of the details of one application: building walls or partitions with interlocking blocks. The problems encountered in this application, the technical and procedural means devised to solve them, the analysis of precision and accuracy, the assessment of trade-off's among different alternatives - all these aspects are outlined here for the reader as an example of a general path of development for various full-size applications of robots in construction. In fact, the authors are now in the process of perfecting two more applications - painting/plastering and tile setting - along a similar path of development.

\section{THE CONCEPT OF BUILDING PARTITIONS/WALLS BY ROBOTS}

Unlike in the case of a multistory hotel, where a standard layout may repeat itself on many floors, the interior partitioning of large floor areas in residential, office, and other types of buildings, has, in many cases, to fit the unique requirements of each tenant or user. In the former case, it may appear advantageous to utilize large prefabricated pieces of partitioning elements, but in the latter cases, a great advantage as to the dimensional flexibility and the tailor-fit interior arrangement, is offered by on-site building of the partitions.

The robotic execution described herein is directed to the latter kind of cases. The partitions are built of lightweight gypsum blocks with interlocking edges, as shown in Fig. 1. They are suitable for both robotic and manual applications, and in fact require man/machine interaction for peripheral problems, even when performed by robots. For the robotic execution it was necessary to break down the task into several simplified 
steps, instead of initiating the manual - rather complex and skillful - fashion of wall building. The robot is greatly inferior to humans in its sensory capabilities, intelligence, and improvization skills, while it is far superior to humans in its physical strength, speed of work, and accuracy. The successful execution of wall-building by TAMIR required, therefore, mutual adaptation between the well-established and proven manual method, and the available robotic technology. The rest of this paper is devoted to a description and a discussion of the following aspects:

* Accuracy analysis,

* Design of the end effector, and

* Calibration and correction of errors.

The paper concludes with the limitations of the system and future plans.

\section{ACCURACY ANALYSIS - ACCUMULATION OF ERRORS}

The final precision of a wall built by TAMIR depends on the accumulation of errors from several sources participating in the building process. To name the major factors:

* The accuracy of the existing adjacent structure, i.e. the precision of columns, walls, floors, ceilings.

* The dimensional accuracy of the building blocks.

* The accuracy of the robot's position and orientation relative to its theoretical location.

* The accuracy of the robotic arm in its pick-and-place operation.

Errors are accumulated from all these (and a few other) sources, and reflected in the final shape and position of the completed wall. If, for example, everything but the robot's position is perfect, the wall will be straight but twisted, while, if everything is perfect but the dimensions of the blocks, the wall may diverge from its intended plane in an arbitrary or in a systematic mode.

\section{KEEPING VERTICALITY OF THE WALL}

Among the aforementioned sources of error, some are more crucial than others. For instance, a slope of only $1 \%$ in the floor, perpendicular to the line marked by the "footprint" of the wall, may cause the entire wall to stay tilted (a $3 \mathrm{~m}$-high wall diverging about $3 \mathrm{~cm}$ at the top). This kind of inaccuracy is unacceptable and must be kept within very close tolerances. In our case, the verticality of the new wall is secured, even if the floor levelling is not perfect, by a "guiding slide" (made of plastic, rubber or wood) which, prior to the entry of the robot on the scene, is attached manually to the 
existing structure in a perfectly vertical position (a in Fig. 2). It simultaneously fulfills two purposes, joining and accuracy: its trapezoid profilation is similar to the interlocking male/female edges of the blocks, thus allowing the first block in each row of the new wall to be connected to the existing structure by this slide. At the same time it serves as a precise reference for the robot to calibrate its vertical axis and define its workframe for the new wall.

\section{LAYOUT PRECISION}

Similar problems of joining and accuracy also exist along the bottom of the wall where it is attached to the floor. Both problems are solved, as in the previous case, by manually fixing "guiding slides", this time to the floor. Thus, when the mobile robot arrives at its theoretical workstation in order to perform its "wall building task", it must first search for two mutually perpendicular slides, one horizontal, the other vertical, whose position is approximately known to the robot. Using accurate $(<1 \mathrm{~mm})$ touch sensors attached to the end of the arm, the robot can define a perfect rectangular workframe for the new wall to be built. Assuming that the entire layout of the partition walls is marked on the floor by such guilding slides, the robot can use them not merely for final calibration at each workstation, but also as leading marks for its navigation. Different properties, such as magnetism, light reflection, coding, etc., can be added to these slides to facilitate their additional task as leading marks.

\section{PICK-AND-PLACE ACCURACY}

Wall building can be considered as a parametric pick-and-place task. However, while in most industrial applications the robot repeats exactly the same movements by taking the same product from the same place to the same new place (e.g. from a fixed position on a conveyor to a fixed position on a machine), in wall building each block is picked from a different place on the pallet, the blocks may have different sizes (e.g. full size and half size), and they are set down at different locations. Most robot manufacturers can guarantee high precision $( \pm 0.1 \mathrm{~mm}$ for our GMF/S700 arm) in the repeatibility of the robot, which is applicable to the former case, but they cannot guarantee even ten times lower accuracy between the theoretical location of the end-effector and its actual position. Our empiric experiments with TAMIR revealed that, at this stage (i.e. with a relatively new robot), the accuracy is around $7 \mathrm{~mm}$ with fully extended arm and maximum payload. 
In wall building we mathematically define a vertical rectangular frame of the wall and determine the building sequence by a parametric computer routine. At the other end of the task we define a de-palletizing routine for picking the blocks. The accumulation of inaccuracies associated with the location of the pallet, the arrangement of the blocks on the pallet, the dimensional tolerances of each individual block, the deflection of the arm, the deflection of the gripper, etc., makes it almost a "mission impossible" to arrive, with each block, at its precise place and in its correct orientation. Although numerous wall segments, of approximately $2 \times 3 \mathrm{~m}$, were successfully built by TAMIR despite all these deficiencies, the process cannot be considered either reliable or safe.

In the following section we shall describe several means which considerably helped to correct these inaccuracies to a tolerable degree.

\section{CALIBRATION OF THE "PICK" PHASE}

When the robot leans towards the stack of blocks organized on the pallet, it is essential to avoid both bumping into the stack with the gripper and premature stoppage, i.e. before reaching the top block. Both incidents are avoided with TAMIR by the following arrangement on the end-effector: The suction pads are connected to the body of the vacuum gripper through sliding pivots, as shown in Fig. 3. The arm is programmed to bring the vacuum gripper to the top of the stack, with its Tool Center Point (TCP) directed downward to the center of the top block and the suction pads hanging from the tool so that their planes are parallel to the face of the top block. From this position the tool moves vertically downward at normal speed until the pads rest on the top block. At this instance a microswitch is opened by the sliding pivot of the pad and operates the vacuum suction and sends a signal to stop the downward motion. There is a delay of $100-250$ milli-seconds until the robot comes to a complete stop, but the additional vertical distance made by the tool is absorbed by the freely sliding pivots of the pads. Meanwhile, the suction pads have established a firm hold on the top block. The arm is instructed to move upwards with the gripped block hanging under the fully extended vacuum pads. At this stage, the block is turned from its horizontal to a vertical position, ready for the next calibration.

\section{TCP CALIBRATION WITH OPTICAL SENSORS}

The block is now firmly held by the vacuum gripper in a vertical position, yet there is no guarantee that the TCP coincides with the center of the block, nor is it guaranteed that the lower edge of the block stays parallel to the floor. The latter error 
is physically corrected with the aid of two optical sensors (Fig. 4) attached to the fixed base (the carriage) of the robot at a known elevation and parallel to one of its horizontal axes. Both sensors are of the transmitter/receiver type. They emit a narrow light beam which is reflected by a close object (up to about $10 \mathrm{~cm}$ distance) and detected by the receiver. If no object exists in front of the sensor, the light beam "gets lost", and no detection is signalled by the receiver of the sensor. These two sensors are utilized in the following manner: The arm passes the block in front of both sensors through a downward motion. In the first instance, when the bottom edge of the block cuts its beam, each sensor is operated separately. The computer "knows" the speed of the downward motion of the block, the horizontal distance between the two sensors, and the time elapsing between the signals of the two sensors, thus it is able to calculate the slope of the bottom edge of the block and send a simple instruction for correction by an appropriate rotation of the last link of the arm. The bottom edge of the block is now roughly levelled and parallel to the floor, yet its center does not necessarily coincides with the TCP of the axm. Now it is moved slowly upwards, to verify that both sensors indeed react simultaneously, i.e. within an acceptable time range. If they do not, a second fine angular correction is performed.

The next corrections of the TCP location are not done physically (as in the previous angular correction) but through software updates.

The block is moved downwards again in slow motion until its bottom edge passes in front of the sensors. The $\mathrm{X}$-axis of the TCP is then redefined to overlap with the X-axis at the center of the block. Finally, the block is lowered about $2 \mathrm{~cm}$ and translated horizontally until its right edge invokes the right sensor (or its left edge invokes the left sensor). At that instance, the $\mathrm{Y}$-axis of the TCP is redefined to overlap with the $Y$-axis of the center of the block.

The calibration of the "pick" phase has been completed, and the block is moved to its intended place in the wall.

\section{THE "PLACE" PHASE}

The previous calibrations ensured that the block can arrive at its theoretical place with sufficiently high precision. However, as explained earlier, its real place may differ from the theoretical one due to a deflection of the arm and the errors accumulated in the completed part of the wall. Consequently, the new block should approach the wall and be laid down very carefully and "sensitively" (otherwise the arm may either move the entire wall and/or move the robot's carriage from its calibrated workstation). Two means have been incorporated in order to achieve a "soft" laydown of the block: (a) the 
suction pads have an additional horizontal degree of freedom, which allows their sliding with respect to the platform of the end-effector. (This degree of freedom had been locked during the entire cycle with the aid of a small piston and released only upon arrival of the block to the vicinity of its final place.) The block is pushed aside against a block set down previously or against the "guiding slide" already located to its left or to its right. Thus a firm contact is ensured among them along their vertical joint. Simultaneously with the release of pressure in the horizontal piston of the tool, the suction force in the vacuum pads is also reduced to allow a vertical gliding of the block with respect to the pads when the arm pushes it downwards about $2 \mathrm{~cm}$ lower than necessary theoretically.

These two means enable the block to be firmly positioned into the corner created by the previous blocks and/or the guiding slides, and actually permit the calibration of the aforementioned pick phase to be less accurate (thus faster). As the positioning of the new block is completed, the vacuum is released, and the arm retreats from the wall to start a new cycle.

\section{LIMITS OF ERROR IN THE BUILT WALL}

The method of building as described so far actually guarantees that at least two edges of the wall (from the bottom and at least one side) will be accurate up to the degree of tolerances allowed between the guiding slides and the interlocking edges of the blocks. Due to these interlocking edges and the "soft" positioning of each block, the total deviation of any block from the theoretical plane of the wall is limited by what little freedom may exist at the joints between neighboring blocks. A theoretical analysis of the accumulated error in average and in extreme cases have led to less than $1 \mathrm{~cm}$ in the worst situations, which was validated by empirical experiments. The detailed analysis is beyond the scope of this paper.

\section{CONCLUSIONS AND FUTURE PLANS}

An entire year was required to bridge the gap between the initial proof of feasibility and a reliable application of the wall building task. It is worthwhile to mention that the wall is still built with a dry method, i.e. without the common adhesive layer of mortar or glue. Experiments are now going on with various fiber-reinforced plasters, which may simultaneously fulfill two objectives, namely as a decorative coating and as a stabilizing agent in lieu of mortar. At the same time other options are also being considered, e.g. dipping each block or spraying it in order to create a thin layer of glue. Upon completion of the wall building task, the other two applications, 
painting/plastering and tile setting, will also be perfected and brought to a satisfactory level of reliability and accuracy.

\section{REFERENCES}

1. Argaman (Stamler), H. Development of a Method for Robotization Planning on the Building Site, Unpublished D.Sc. Dissertation (A. Warszawski, supervisor), Technion, I.I.T., Israel, October 1989.

2. Rosenfeld, Y. and Berkovitz, S. "Automation of Existing Tower Cranes - Economic and Safety Examination", Proc. of the 6th International Symposium on Automation and Robotics in Construction, San Francisco, U.S., June 1989, pp. 420-426.

3. Rosenfeld, Y. and Berkovitz, S. Automation of Tower Cranes at Construction Sites, Research report No. 017-438, National Building Research Institute, Technion, Haifa, 1990.

4. Rosenfeld, Y., Warszawski, A. and Zajicek, U. "Robotic Performance of Interior Finishing Works: Development of Full Size Applications", Proc. of the 7th Int. Symp. on Automation and Robotics in Construction, Bristol, UK, June 1990.

5. Warszawski, A. "Application of Robotics to Building Construction", Civil Engineering and Construction Laboratory, Carnegie Mellon University, 1984.

6. Warszawski, A. Industrialization and Robotics in Building, Harper and Row Publishers, NY, 1990.

7. Warszawski, A. and Argaman, M. "Teaching Robotics in Building", Proc. of the Fourth International Symposium on Robotics and Artificial Intelligence in Building Construction, Haifa, Israel, June 22-25, 2987, pp. 245-258.

8. Warszawski, A. and Navon, R. "Robot for Interior Finishing Works". Accepted for publication in ASCE J. of Constr. Engrg. \& Management, Sept. 1991. 


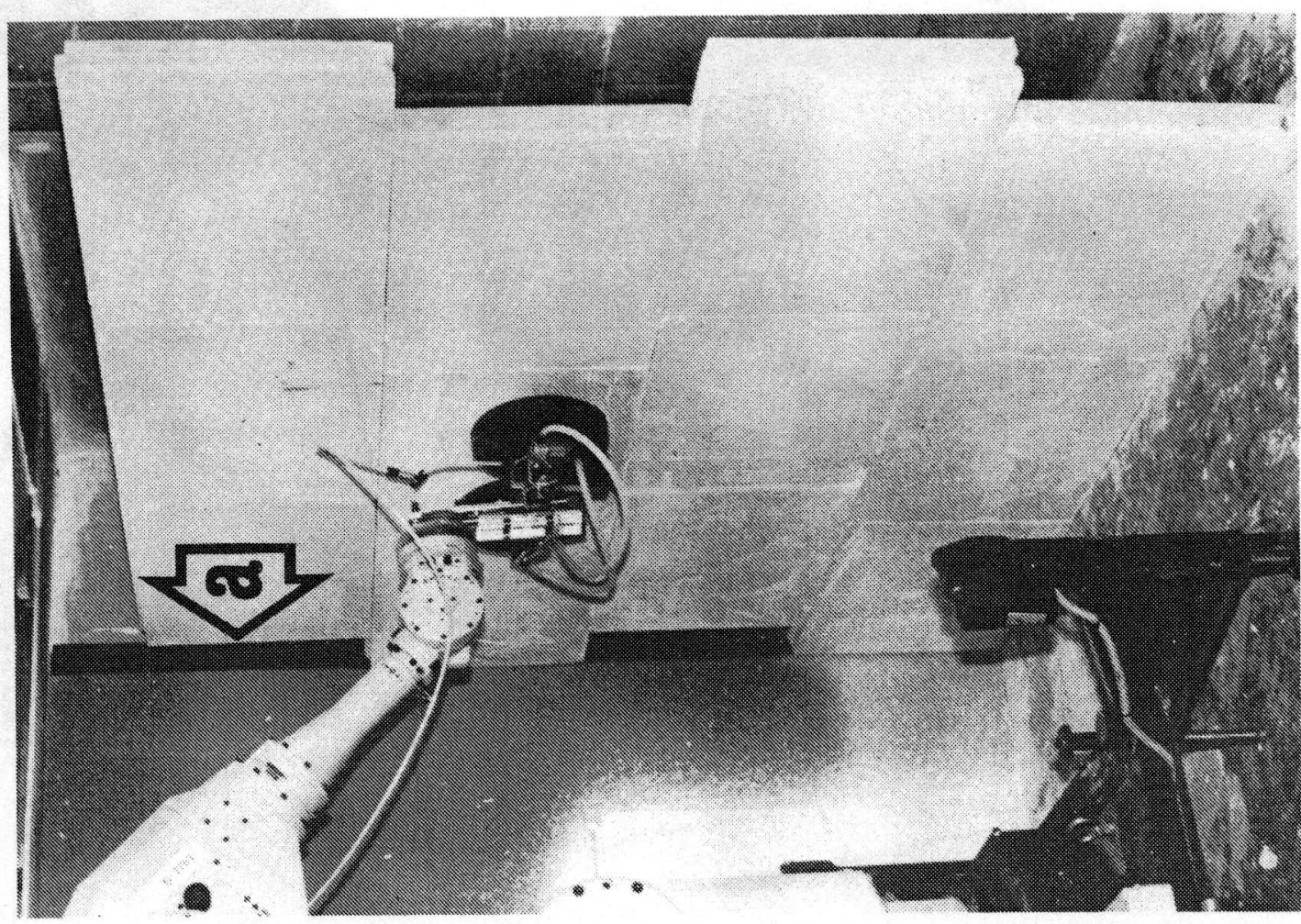

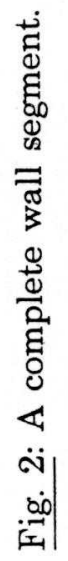

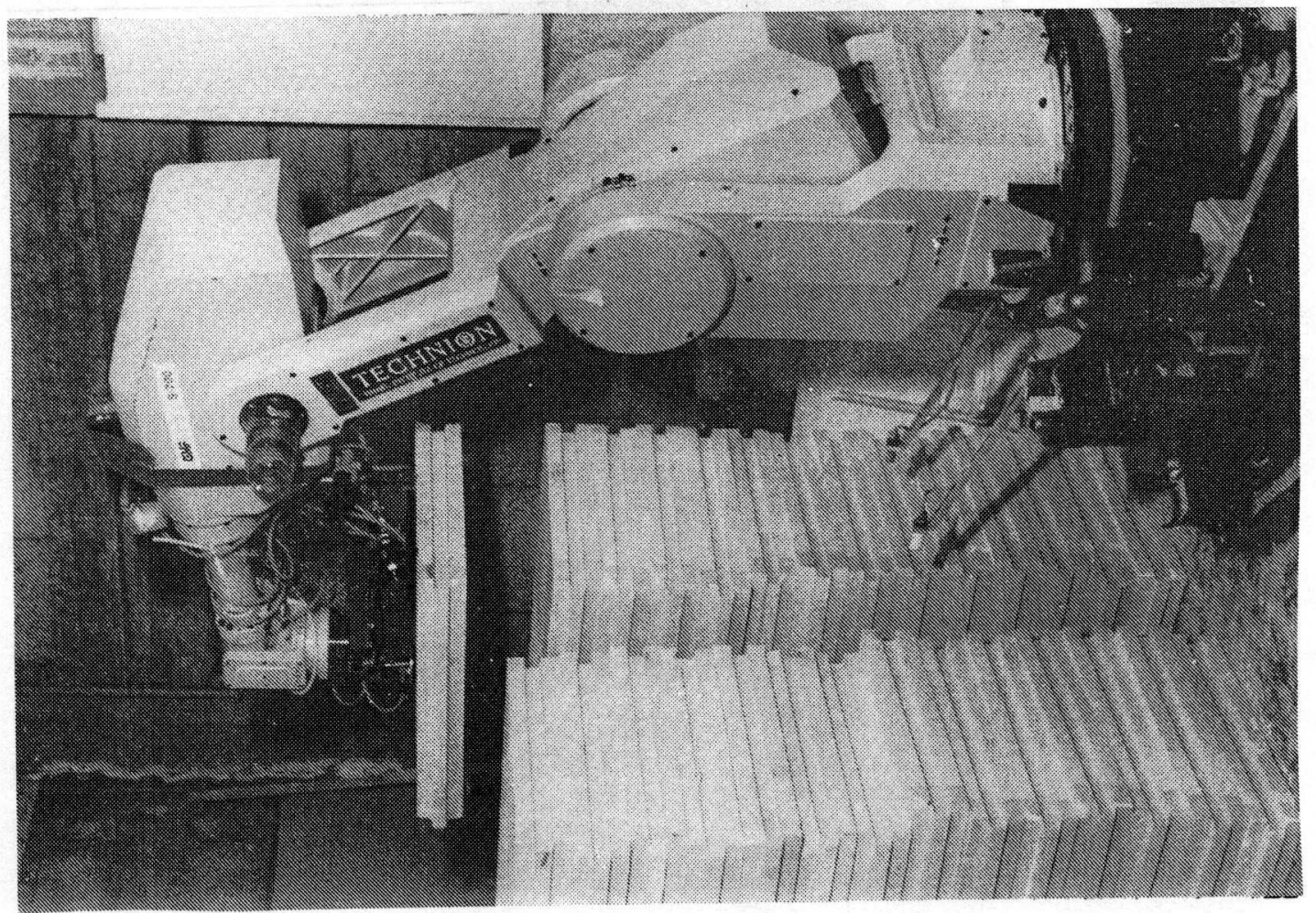

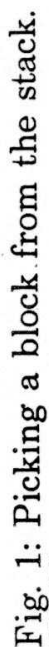




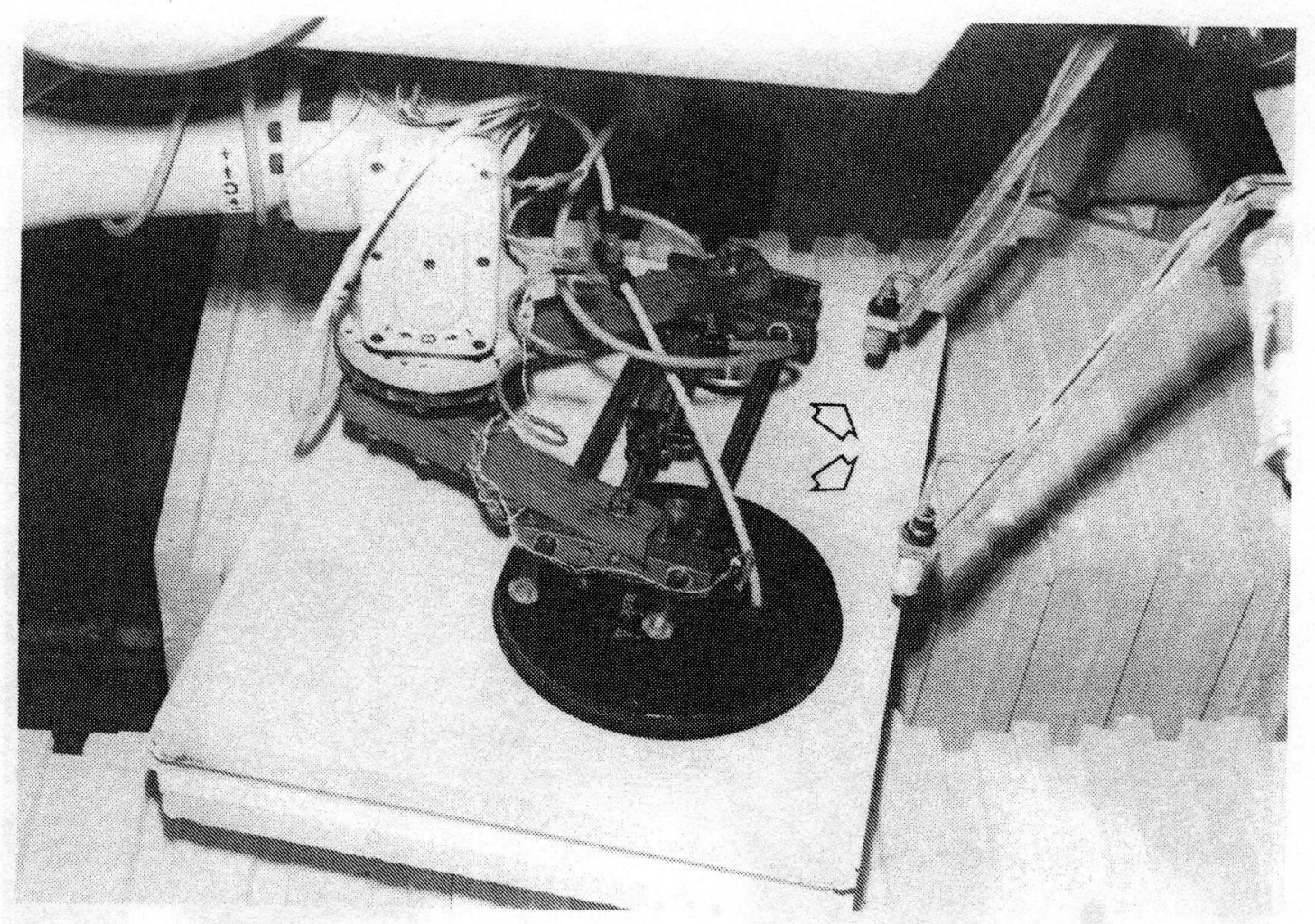

О

起

둥

ํㅠㄱ

유

क्.

ฮี

융

है

守|

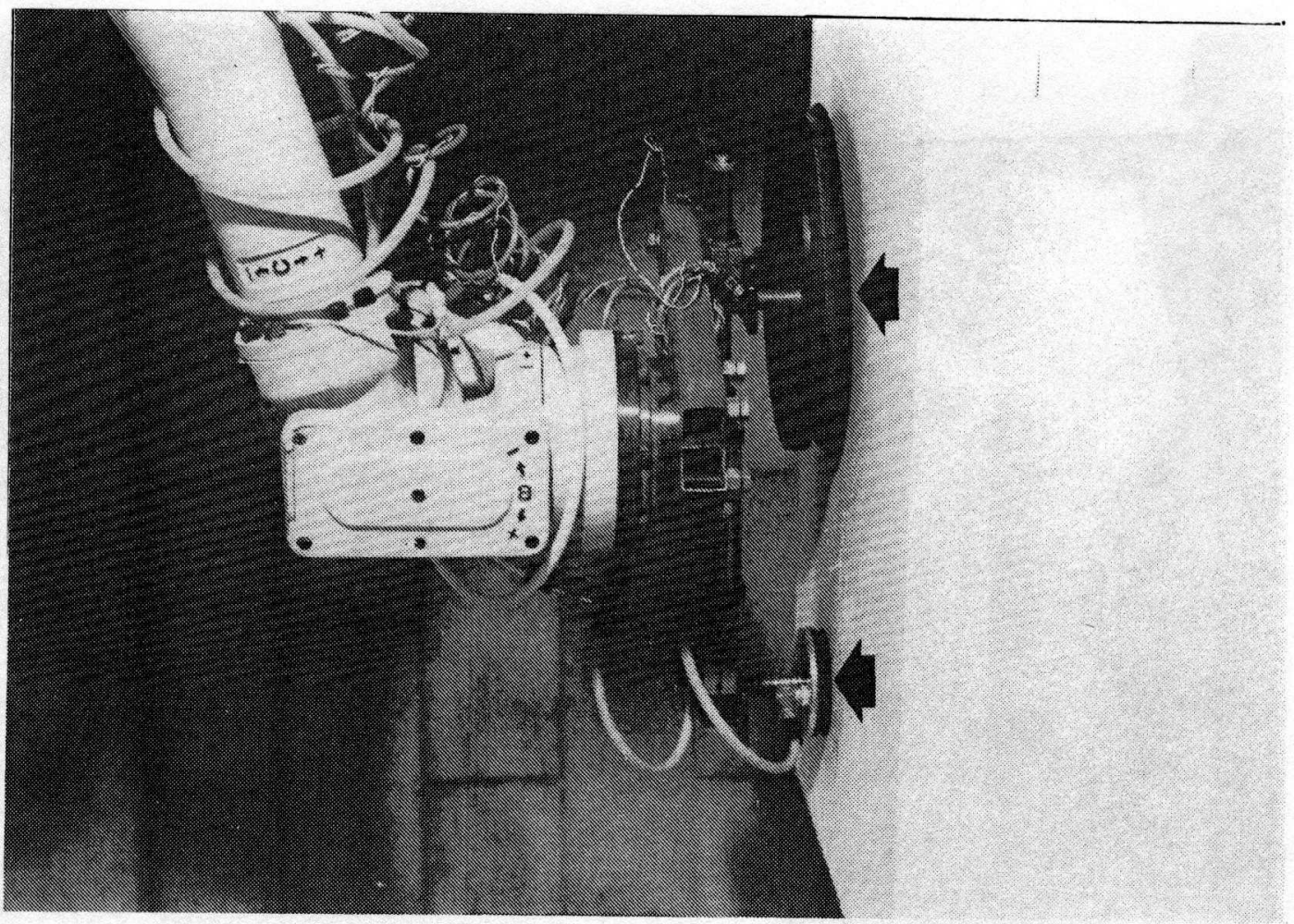

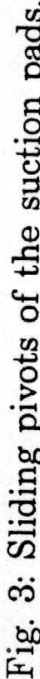

